\title{
Research on Audio Digital Watermark and realization in MATLAB
}

\author{
Biqing $\mathrm{Li}^{1, \mathrm{a}}, \mathrm{Zhao} \mathrm{Li}^{* 2, \mathrm{~b}}$ \\ ${ }^{1}$ College of Mechanical and Electronic Engineering, Hezhou university, Hezhou \\ Guangxi 542899, China; \\ ${ }^{2}$ Management EngineeringDepartment, \\ Guangxi vocational andtechnicalcollege ofcommunications,Liuzhou Guangxi \\ 530000, China; \\ ajanliful@163.com,,229292710@qq.com
}

\begin{abstract}
Based on improved algorithm of DCT(discrete cosine transform), the Audio Digital Watermarks is embeded and detected in this paper.The watermark embedding process is simulated by MATLAB was described in this paper. The experimental results shows that the watermarks by this algorithm has good stability and non sense. Audio watermarking with MATLAB software can meet the real-time requirements and can better protect the copyright information.
\end{abstract}

Key words: Audio watermarking; DCT; watermark embedding; watermark extracting

\section{Introduction}

In order to prevent piracy and protect copyright, a lot of people are embedding watermark in the works. To embed watermark in audio is relatively difficult, it's not easy to identify only through the ear. The human ear system is sensitive to additive noise.It is difficult to detect the robustness and the non sense of the watermark by embedding the watermark in the time domain. For example,watermark can be embedded by using the masking effect of human ear system and non-sensitive properties of the human ear system to absolute phase.

\section{Audio digital watermark embedding}

The steps of audio watermark embedding are as follows:

Firstly, using the discrete cosine transform to segment the Audio data Ae embedding with watermark .

$$
\mathrm{De}=\operatorname{DCT} \mathrm{Ae}=\{\operatorname{De}(\mathrm{k})=\operatorname{DCT} \operatorname{Ae}(\mathrm{k})\} 0 \leq \mathrm{k}<\mathrm{M} 1 \times \mathrm{M} 2
$$

$\operatorname{De}(\mathrm{k})=\operatorname{de}(\mathrm{k})(\mathrm{m}), 0 \leq \mathrm{m}<\mathrm{N}$ is the $m_{\text {coefficient during the the discrete cosine }}$ transform $\mathrm{De}(\mathrm{k})$ of $k$ Audio data segment.

Secondly, the DCT transform is used to determine the intermediate frequency 
coefficients of the digital audio signal, which is used to embed the corresponding elements $\mathrm{Vp}(\mathrm{k})$ in the sequence $\mathrm{Vp}$. The number of audio data segment data $\mathrm{Ae}(\mathrm{k})$ is $N$, the change result $\operatorname{De}(\mathrm{k})$ of DCT with $N$ DCT transform coefficients, for the DC component $\operatorname{de}(\mathrm{k})(0)$ is the zero DCT coefficients of $\mathrm{de}(\mathrm{k})(0)$, AC component of low frequency to high frequency isN-1 DCT coefficients. In order to improve the robustness of the embedded watermark, usually we choose the low frequency AC component $\operatorname{de}(\mathrm{k})(1)$ as intermediate frequency coefficient, if choose $\mathrm{de}(\mathrm{k})(2)$ as intermediate frequency coefficient, that $\mathrm{m}_{\mathrm{w}}=2$.

Thirdly,modify the intermediate frequency coefficient $\operatorname{de}(\mathrm{k})\left(\mathrm{m}_{\mathrm{w}}\right)$ and embed it into element $\mathrm{Vp}(\mathrm{k})$ of sequence $\mathrm{Vp}$.

$$
d e^{\prime}(k)=d e(k)(m)(1+\partial v p(k)), m=m w
$$

$\alpha$ is the ratio coefficient, which can control the position of intermediate frequency coefficient. If the $\alpha$ value is too small,the watermark robustness will be worse; if the value is too large, the actual use value of the original digital audio signal is too low. Before the digital audio watermark is obtained, the audio watermark is a watermark which is a discrete cosine transform.

$$
A e^{\prime}=I D C T\left(D e^{\prime}\right)=\left\{\operatorname{IDCT}\left(D e^{\prime}(k), 0 \leq k<\left(M_{1} \times M_{2}\right)\right\}\right.
$$

Finally,we put the results obtained into the formula and complete the embedding.

$$
A_{w}=A_{r}+A e^{\prime}
$$

\section{Audio digital watermark extracting}

Firstly, the original digital audio $\operatorname{signal} A$ and the digital audio signal $A s$ to be detected are segment processed.

$$
\mathrm{A}=\mathrm{Ae}+\mathrm{Ar}
$$

Secondly, the watermark part $\mathrm{Ae}_{\text {of }}$ the original audio signal will be used to embed and the watermark part Ase of the audio signal to be detected are discrete cosine transformed .

$$
\operatorname{De}=\operatorname{DCT}(\mathrm{Ae})=\operatorname{De}(\mathrm{k})=\operatorname{DCT}(\operatorname{Ae}(\mathrm{k})), 0<\mathrm{k}<\mathrm{M} 1 \times \mathrm{M} 2
$$

The obtained signal is extracted from the discrete cosine transform domain, and the 
watermark sequence is obtained by the formula (6)

$$
\mathrm{x}=1 /\left(\alpha \times \operatorname{de}(\mathrm{k})\left(\mathrm{m}_{\mathrm{w}}\right)\right)\left(\operatorname{dse} \mathrm{e}^{\prime}(\mathrm{k})\left(\mathrm{m}_{\mathrm{w}}\right) \operatorname{de}(\mathrm{k})\left(\mathrm{m}_{\mathrm{w}}\right)\right)
$$

Thirdly, the extraction sequence $\mathrm{Vsp}^{\mathrm{s}}$ of fake random sequence reverse sort (i.e. anti scrambling), to get a sequence $\mathrm{Vs}$ of watermark extraction.

$$
\text { Vs }=\text { InversePermute }(\text { Vse })=\{\mathrm{Vs}(\mathrm{k}), 0<\mathrm{k}<(\mathrm{M} 1 \times \mathrm{M} 2)
$$

Fourthly, Two-dimensional transform processing the Vs, getting the image of two-dimensional sequence, so as to successfullyextract the watermark .

In order to prevent the results from being affected by other factors, the normalized correlation coefficient is used to evaluate the similarity between the original watermark and the extracted watermark, as shown in the formula (9):

$$
\rho(W, W s)=\frac{\sum_{i=1}^{M_{1}} \sum_{j=1}^{M_{2}} W(i, j) W s(i, j)}{\sqrt{\sum_{i=1}^{M_{1}} \sum_{j=1}^{M_{2}} W(i, j)^{2}} \sqrt{\sum_{i=1}^{M_{1}} \sum_{j=1}^{M_{2}} W s(i, j)^{2}}}
$$

When the image resolution is assumed to be $32 \times 32$, dividing them into $4 * 4=16$ blocks. When the watermark image resolution is assumed to be $64 \times 64$, and then dividing them into 64 blocks, you can get a piece of the size of 64 . Then we can remove the 16 intermediate frequency coefficients of the image and watermark blocks

\begin{tabular}{|c|c|c|c|c|c|c|c|c|c|c|c|}
\hline 0 & 1 & 5 & 6 & 14 & 15 & 27 & 28 & & & & \\
\hline 2 & 4 & 7 & 13 & 16, & 26 & 29 & 42 & & & & \\
\hline 3 & 8 & 12 & 817 & 25 & 30 & 41 & 43 & 14 & 15 & 13 & 16 \\
\hline 9 & 11 & 18 & 24 & 31 & 40 & 44 & 53 & 12 & 17 & 25 & 18 \\
\hline 10 & 19 & 23 & 32 & 39 & 45 & 52 & 54 & 24 & 19 & 23 & 20 \\
\hline 20 & 22 & 30 & 38 & 46 & 51 & 55 & 60 & 22 & 21 & 34 & 35 \\
\hline 212 & 34 约 & 37 & 47 & 50 & 58 & 59 & 61 & & & & \\
\hline 35 & 36 & 48 & 48 & 57 & 58 & 62 & 63 & & & & \\
\hline
\end{tabular}
embed into watermark. Theshadows in the left picture $8 * 8$ block coefficient part is being pumped out of . As shown in figure 1 .

Figure 1 Selecting 4 x 4 intermediate position coefficient in DCT area

\section{MATLAB simulation}

In the experiment,the audio sampling rate we selected is WAV format $44.1 \mathrm{kHz}$ voice signal, the watermark image is the gray image of 16bit. Thewaveform of original audio carrier work, and the audio work waveform after embedded watermark as shown in figure 2: 


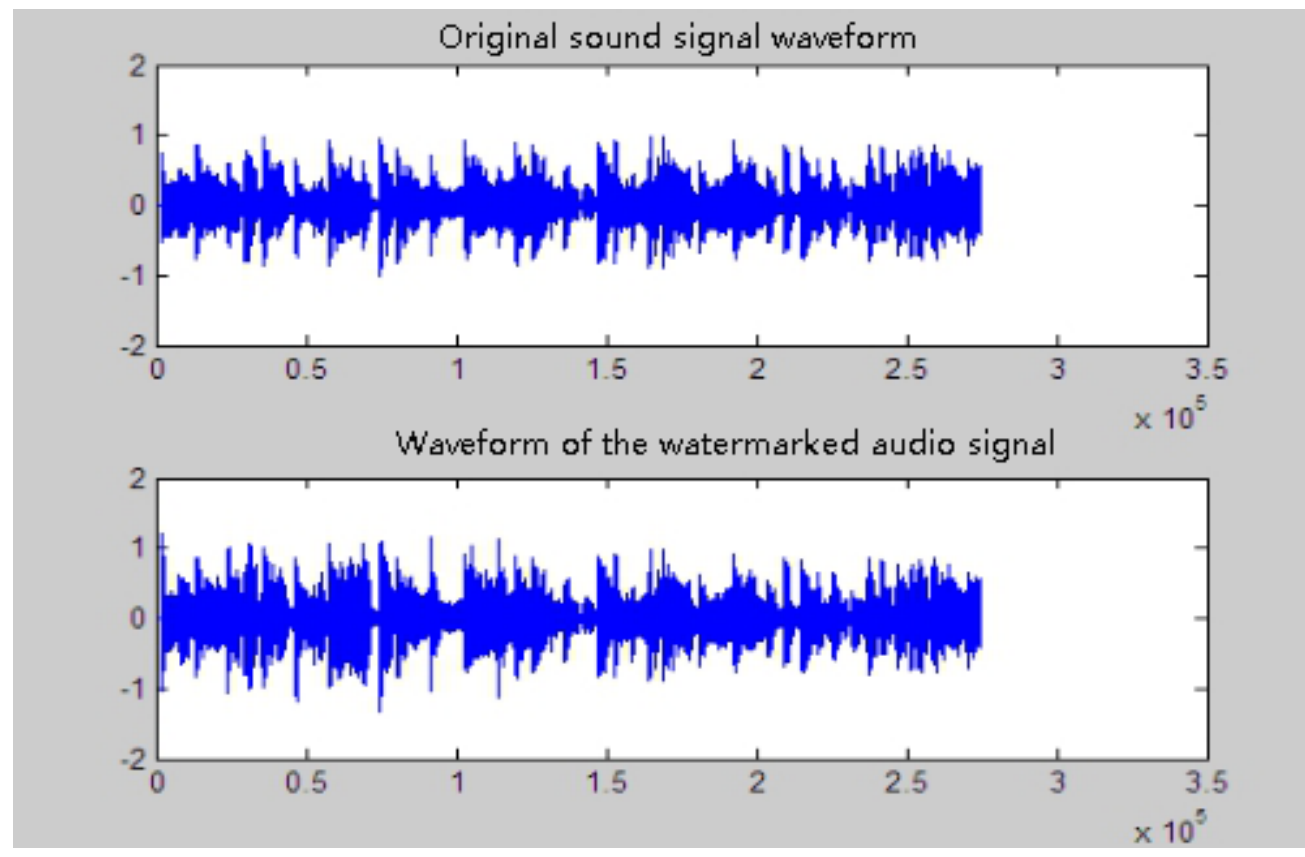

Fig. 2 The audio waveform before and after embedding watermark

By comparing the audio waveform before and afterembedding watermark, the front parts of the 2 waveform are different. This is because that the watermark is added in the previous section,the waveform diagram has changed.

\section{Conclusions}

This design uses the MATLAB software programming, the discrete cosine transform to carry on the watermark embedding and the extracting. Discrete cosine transform (DCT) has better sensitivity and concentrating energy, which is very effective to audio signal processing. The improved algorithm DCT intermediate frequency watermark embedding algorithm can be obtained through the DCT transform coefficients to determine the embedding place.

\section{Acknowledgement}

This work is supported the following fund :

2016 The project of improving the basic ability of young teachers in Colleges and universities in Guangxi:"Design and development of electronic commerce platform of agricultural products based on Semantic Technology"(No,KY2016YB455).

2016 Guangxi higher education teaching reform project: "Exploration on the cultivation mechanism of the teaching characteristics of Communication Engineering Specialty Based on ZTE ICT education platform"

2015 college students' innovative training program: "Research on the application of value added travel experience in the mobile terminal of the 'ethnic custom travel' in Guangxi" (No201511838070);\&\& “The design and development HeYuanTong Campus Mobile Phone APP based on Android" (No 201511838034).

2015 Teaching case project construction projectof hezhou university: "Tourist positioning and guiding system project of Huangyao scenic";\&\& "Development and 
application of the information management system of 'Huang Yao Tong"'.

Project of scientific research and technology development project of Hezhou: "Design and implementation of agricultural products e-commerce platform based on Semantic Technology"(No,Hekeneng 1506006)

Scientific research project of hezhou university:"Research and development of E-government platform based on mobile terminal" (No2015ZZZK03);

Reform in Educationproject of hezhou university: "Internet of things"(No hzxytszy201501); "Research on application personnel training model of Communications for SMEs"(Nohzxyjg201525);

Master degree discipline construction scientific research and Cultivation Project: "Research on the social work service of the teenagers' Network Addiction"(No 2015SHGZ005) \&\&"Research on the intelligent development of rural tourism in Guangxi based on the Internet of things" (No2015MTA16);

\section{Reference}

[1].Zheng SQ, DZ; Qiang, Z ; Li, BQ. Design and Implementation of Supermarket Personnel Management System Based On Java. In: Chen PZ, S, editor. 2nd International Conference on Education, Management and Computing Technology (ICEMCT); Tianjin, PEOPLES R CHINA: ATLANTIS PRESS; 2015. p. 1724-7.

[2].Li BG, WL; Zheng, SY ; Yue, XG. OPTIMISATION DESIGN OF CORN PRECISION SEEDER BASED ON MULTI-ROUTE AND MULTI-CHANNEL CONTROL[J]. JOURNAL OF THE BALKAN TRIBOLOGICAL ASSOCIATION. 2015,21(4A): 1215-23.

[3].Zheng SG, Weili; Li, Biqing;. Social Work in Teen Addiction Correction Services Research Under the New Situation. In: Wang JX, P, editor. 4th National Conference on Electrical, Electronics and Computer Engineering (NCEECE); Xian, PEOPLES R CHINA: ATLANTIS PRESS; 2016. p. 252-5.

[4].Zheng SG, WL ; Li, BQ. Digital Display Design of Ethnic Clothing of Nanling. In: Liang ZL, X, editor. 4th International Conference on Mechatronics, Materials, Chemistry and Computer Engineering (ICMMCCE); Xian, PEOPLES R CHINA: ATLANTIS PRESS; 2015. p. 2805-8.

[5].Shiyong Zheng WG, Jing Wang,Biqing Li,Deze QIN. Analysis of Internet of Things Talent Training and Curriculum System Innovation. 3th International Conference on Education,Management and Computiong Technology; HangZhou,China: ATLANTIS PRESS; 2016. p. 957-60. 